\title{
Reconstruction of High-Resolution Computed Tomography Image in Sinogram Space
}

\author{
Osama A. Omer \\ Aswan faculty of Engineering, Aswan University, Aswan, Egypt \\ Email: omer.osama@aswu.edu.eg \\ Received: June 30, 2019. Revised: August 4, 2021. Accepted: September 11, 2021. Published: November 27, 2021.
}

\begin{abstract}
An important part of any computed tomography (CT) system is the reconstruction method, which transforms the measured data into images. Reconstruction methods for CT can be either analytical or iterative. The analytical methods can be exact, by exact projector inversion, or non-exact based on Back projection (BP). The BP methods are attractive because of thier simplicity and low computational cost. But they produce suboptimal images with respect to artifacts, resolution, and noise. This paper deals with improve of the image quality of BP by using super-resolution technique. Super-resolution can be beneficial in improving the image quality of many medical imaging systems without the need for significant hardware alternation. In this paper, we propose to reconstruct a high-resolution image from the measured signals in Sinogram space instead of reconstructing low-resolution images and then post-process these images to get higher resolution image.
\end{abstract}

\section{INTRODUCTION}

In the areas of medical diagnostics and non-destructive testing, it is of great interest to be able to capture images of the interior of objects. One common technique to accomplish this feat is known as Computed Tomography (CT), which invented in 1972 [1]. A CT scanner uses digitally sampled $\mathrm{X}$-ray images acquired in multiple directions to calculate crosssectional images of the X-ray attenuation of an object.

An important part of any CT system is the reconstruction method, which transforms the measured data into images. Reconstruction methods for CT can be either analytical or iterative. Analytical methods can be either exact and non-exact. Exact methods are based on exact inversion of the projector in the continuous domain. Although efficient exact methods exist they are currently not found in clinical use. Instead, manufacturers of clinical CT systems employ non-exact methods, based on Backprojection (BP) methods. Due to approximations in the derivation of these methods, reconstruction results are contaminated by artifacts. In return, non-exact methods are computationally less demanding, simpler to implement, and offer a better dose utilization than exact methods [1], [2].

On the other hand, high-resolution images reveal more information than low-resolution images, which therefore ease disease diagnosis and detection. Early, fast, and accurate detection of imaging biomarkers of the onset and progression of diseases is of great importance to the medical community since early detection and intervention often results in optimal treatment and recovery. However, earlier biomarkers of disease onset are often critically smaller or weaker in contrast compared to their corresponding features in the advanced stages of disease [3].
One way to increase the images resolution is to physically reduce the pixel size and therefore increase the number of pixels per unit area. However, a reduction of pixel size causes degradation in the image quality. Instead of altering the sensor manufacturing technology, digital image processing methods to obtain an HR image from low-resolution (LR) observations have been investigated by many researchers [3]-[10]. Other researchers use interpolation techniques to enhance the quality of the medical images [11], however, the interpolation will not add new information to the under-sampled signals. In practice, it is common to take multiple scans of the same subject and average them to improve the signal-to-noise ratio (SNR) of the final image [12]. Also, such an approach makes no improvement in image resolution. Super-Resolution (SR) algorithms are an interesting way to increase the resolution of images. They are based on the fact that, by combining various low resolution (LR) and highly correlated images, it is possible to obtain a high resolution image (HR) by using the information from different images.

The goal in this paper is to enhance the resolution for CT image using multi-images super-resolution technique. Unlike the existing super-resolution methods that are usually done as a post-process, we propose to solve the SR problem in the sinogram space.

\section{CT RECONSTRUCTION}

There are two main types of CT reconstruction techniques. The first type is a Fourier-based technique, such as Filtered backprojection and linogram. The other type is iterative-based technique. The iterative based technique is algebraic and statistical approaches [1]. Simply, these methods are trying to get the closest approximation of the density function of the object by using iterative techniques. Since filtered backprojection is the most used algorithm in modern CT, we will adopt it in the reconstruction of HR CT images.

There are three main ways for CT reconstuctions using backprojection, namely, reconstruction of pencil-beam, fanbeam and conebeam CT. For simplicity and without loss of generality, we will use pencil-beam reconstruction.

\section{A. The Radon Transform}

We will focus on explaining the Radon transform of an image function and discussing the inversion of the Radon transform in order to reconstruct the image [1].

We will discuss only the 2D Radon transform, although some of the discussion could be readily generalized to the 3D 
Radon transform. The Radon transform (RT) of a distribution $f(x, y)$ is given by

$$
p(\zeta, \phi)=\int f(x, y) \delta(x \cos \phi+y \sin \phi-\zeta) d x d x
$$

where $\delta$ is the Dirac delta function and $x, y, \zeta$, and $\phi$ are the coordinates. The task of tomographic reconstruction is to find $f(x, y)$ given knowledge of $p(\zeta, \phi)$.

\section{B. Backprojection}

Mathematically, the backprojection operation is defined as:

$$
f_{B P}(x, y)=\int_{0}^{\pi} p(x \cos \phi+y \sin \phi, \phi) d \phi
$$

Geometrically, the backprojection operation simply propagates the measured sinogram back into the image space along the projection paths.

\section{RESOLUTION ENHANCEMENT OF CT IMAGES}

\section{A. Super-Resolution Problem Description}

The multi-images super-resolution problem can be simply described, in matrix-vector notation, as [9], [10]

$$
\underline{Y}_{k}=\mathbf{D}_{k} \mathbf{B}_{k} \mathbf{W}_{k} \underline{X}+\underline{V}_{k}, \quad k=1: N
$$

Where $\mathbf{W}_{\mathbf{k}}$ is the geometric motion operator between the HR frame $\underline{X}$ and the $K-t h$ LR frame, $\underline{Y}_{k}$. The system point spread function (PSF) is modelled by the sparse matrix $\mathbf{B}_{\mathbf{k}}$, and $\mathbf{D}_{\mathbf{k}}$ is a sparse matrix that represents the decimation operator. The vector $\underline{V}_{k}$ is the system noise and $N$ is the number of available LR images. For convenience, concatenate all the measurements in one vector as follows

$$
\underline{Y}=\mathbf{H} \underline{X}+\underline{V},
$$

From many available estimators, which estimate a HR image from a set of noisy LR images, one may choose to find the most probable $\mathrm{X}$, given the measurements, $\mathrm{Y}$, that is the maximum a-posterior probability(MAP), which can be described as to maximize

$$
\operatorname{Pr}\{\underline{X} \mid \underline{Y}\}
$$

Where $\operatorname{Pr}\{\underline{X} \mid \underline{Y}\}$ is the probability of the HR image, $\underline{X}$, given the measurements $\underline{Y}$

$$
\operatorname{Pr}\{\underline{X} \mid \underline{Y}\}=\frac{\operatorname{Pr}\{\underline{Y} \mid \underline{X}\} \operatorname{Pr}\{\underline{X}\}}{\operatorname{Pr}\{\underline{Y}\}}
$$

Therefore, the solution for the maximum a-posteriori probability is described as

$$
\begin{aligned}
\underline{\hat{X}}_{M A P} & =\operatorname{ArgMax}_{\underline{X}} \operatorname{Pr}\{\underline{X} \mid \underline{Y}\} \\
& =\operatorname{ArgMax}_{\underline{X}} \operatorname{Pr}\{\underline{Y} \mid \underline{X}\} \operatorname{Pr}\{\underline{X}\}
\end{aligned}
$$

By assuming Gaussian distribution for noise and Gibbs distribution with some energy function $A(X)$ for the prior information, we get

$$
\operatorname{Pr}\{\underline{X}\}=\text { Const. } \exp (-A(\underline{X}))
$$

Then

$$
\begin{aligned}
\underline{\hat{X}}_{M A P} & =\operatorname{ArgMax}_{\underline{X}} \operatorname{Pr}\{\underline{Y} \| \underline{X}\} \operatorname{Pr}\{\underline{X}\} \\
& =\operatorname{ArgMin}_{\underline{X}} \sum_{k=1}^{N}\left\|\mathbf{D B W}_{k} \underline{X}-\underline{Y}_{k}\right\|^{2}+\lambda A(\underline{X})
\end{aligned}
$$

The last term $A(\underline{X})$ represents the regularization term and $\lambda$ represents the regularization parameter. There are many choices for $A(X)$, depending on the priori information, including Gaussian prior

$$
A(\underline{X})=\|\underline{X}\|^{2}
$$

And the bilateral prior

$$
\left.A(\underline{X})=\sum_{n=-P}^{P} \sum_{m=-P}^{P} a_{m n} \dot{(X}-\mathbf{S}_{x}^{n} \mathbf{S}_{y}^{m} \underline{X}\right)
$$

where $\mathbf{S}_{\mathbf{x}}^{\mathbf{n}}$ is a shifting operator by $n$ pixels in $x$ direction [9]. There are two types of noise exist in this imaging model, namely, additive noise (usually assumed to be Gaussian) and registration noise (registration error), which can be assumed as Laplacian noise [9]. Based on the modeling of the total noise, the data fidelity term will change.

- In case of Gaussian Noise assumption:

$$
J_{M A P}(\underline{X})=\sum_{k=1}^{N}\left\|\mathbf{D}_{k} \mathbf{B}_{k} \mathbf{W}_{k} \underline{X}-\underline{Y}_{k}\right\|_{2}^{2}+\lambda A(\underline{X})
$$

- In case of Laplacian Noise assumption:

$$
J_{M A P}(\underline{X})=\sum_{k=1}^{N}\left\|\mathbf{D}_{k} \mathbf{B}_{k} \mathbf{W}_{k} \underline{X}-\underline{Y}\right\|_{k}^{1}+\lambda A(\underline{X})
$$

Where $\|\cdot\|_{1}^{1}$ is the L1-norm.

\section{B. Two-Steps-Based CT Super-Resolution}

Resolution enhancement of medical image is usually done in two steps, namely, image reconstruction step and resolution enhancement step. The image reconstruction can be modeled in matrix-vector multiplication as [12]

$$
\underline{U}=\mathbf{S} \underline{\mu}+\underline{\eta}
$$

Where $\mathbf{S}$ is the reconstruction matrix that relates the measured signal, $\underline{U}$, with the pixel values, $\mu$ and $\eta$ is the additive noise. As a reverse problem, estimating $\mu$ can be done by minimizing

$$
J(\underline{\mu})=\|\mathbf{S} \underline{\mu}-\underline{U}\|_{2}^{2}+\lambda\|\underline{\mu}\|_{2}^{2}
$$

After reconstructing N LR images, these LR images can be fused to get a high-resolution image. The resolution enhancement problem is therefore described as

$$
\underline{\mu}_{k}=\mathbf{D}_{k} \mathbf{B}_{k} \mathbf{W}_{k} \underline{X}+\underline{V}_{k}, \quad k=1: N
$$

which can be solved by minimizing the cost function

$$
J(\underline{X})=\sum_{k=1}^{N}\left\|\mathbf{D}_{k} \mathbf{B}_{k} \mathbf{W}_{k} \underline{X}-\underline{\mu}_{k}\right\|_{2}^{2}+\lambda(\underline{X})\|\underline{\mathbf{C} X}\|_{2}^{2}
$$

Where $\mathbf{C}$ is a sparse matrix representing a high-pass filter operator, the last term is the Tikhonov regularization. 


\section{Reconstruction of High-Resolution CT}

Instead of performing resolution enhancement in two steps as shown in the previous Section, substituting from (14) into (12) leads to

$$
\begin{aligned}
\underline{U}_{k} & =\mathbf{S D}_{k} \mathbf{B}_{k} \mathbf{W}_{k} \underline{X}+\underline{\eta}_{k}+\mathbf{S} \underline{V}_{k}, \quad k=1: N \\
& =\mathbf{S D}_{k} \mathbf{B}_{k} \mathbf{W}_{k} \underline{X}+\underline{E}_{k} \quad k=1: N
\end{aligned}
$$

With

$$
\underline{E}_{k}=\underline{\eta}_{k}+\mathbf{S} \underline{V}_{k}
$$

Then the HR CT reconstruction problem, by assuming that the total contaminating noise has Laplacian distribution, is simplified to minimize

$$
J_{1}(\underline{X})=\sum_{k=1}^{N}\left\|\mathbf{S D B}_{k} \mathbf{W}_{k} \underline{X}-\underline{U}_{k}\right\|_{1}^{1}+\lambda(\underline{X})\|\mathbf{C} \underline{\mathbf{X}}\|_{\mathbf{1}}^{\mathbf{1}}
$$

Where $\underline{X}$ is the HR image. $\underline{U}_{k}$ is the measured signal. Without oss of generality, we assume that the blurring operator is the same for all images, then $\mathbf{B}_{\mathbf{k}}=\mathbf{B}$ and that the downsampling operator is the same for all images, then $\mathbf{D}_{\mathbf{k}}=\mathbf{D}$. Also considering that the system function in MRI can be representing by Radon transformation, then $\mathbf{S}=\mathbf{R}$. Then (19) becomes

$$
J_{1}(\underline{X})=\sum_{k=1}^{N}\left\|\mathbf{R D B} \mathbf{W}_{k} \underline{X}-\underline{U}_{k}\right\|_{1}^{1}+\lambda(\underline{X})\|\mathbf{C} \underline{X}\|_{1}^{1}
$$

Where the regularization parameter, $\lambda(\underline{X})$, can be calculated adaptively as a function of the cost function as [10], then

$$
\lambda(\underline{X})=\frac{\sum_{k=1}^{N}\left\|\mathbf{R D B} \mathbf{W}_{k} \underline{X}-\underline{U}_{k}\right\|_{1}^{1}}{\frac{1}{\gamma}-\|\mathbf{C} \underline{\mathbf{X}}\|_{1}^{\mathbf{1}}}
$$

With

$$
\frac{1}{\gamma}=2 \sum_{k=1}^{N}\left\|\underline{U}_{k}\right\|_{1}^{1}
$$

As stated in Section III-A, the data fidelity term and therefore the cost function depend on the noise model. The total contaminating noise $\underline{E}_{k}\left(\underline{E}_{k}=k+\mathbf{S} \underline{V}_{k}\right)$ is a result of many sources, including thermal noise, and registration error.

\section{Simulation Results}

\section{A. Data Sets}

In this section we present experiments illustrating the performance of the proposed algorithm. The experiments are conducted on Phantom sequence. This sequence contains 4 measured signals, in Sinogram space, to increase resolution by factor $2 \times 2$. The size of each LR image is $256 \times 256$.

\section{B. Experiment Setup}

To test the efficiency of the proposed HR CT reconstruction algorithm, we compared it with the reconstructed LR image. The cost function in (20), is solved iteratively using steepest decent as

$$
\begin{aligned}
\underline{\hat{X}}_{n+1} & =\underline{\hat{X}}_{n} \\
& -\beta \sum_{k=1}^{N} \mathbf{W}_{\mathbf{k}}^{\mathbf{T}} \mathbf{B}^{\mathbf{T}} \mathbf{D}^{\mathbf{T}} \mathbf{R}^{\mathbf{T}} \operatorname{sign}\left(\mathbf{R D B} \mathbf{W}_{\mathbf{k}} \underline{\hat{\mathbf{X}}}_{\mathbf{n}}-\underline{\mathbf{U}}_{\mathbf{k}}\right) \\
& +\lambda \mathbf{C}^{\mathbf{T}} \operatorname{sign}\left(\mathbf{C} \underline{\hat{X}}_{\mathbf{n}}\right)
\end{aligned}
$$

The setup of these experiments is as follow, resolution enhancement factor $=2, \beta=4, \lambda=0.02$, and maximumiteration $=10$. The number of LR images used is 4 . The relative motions of the generated LR phantom images, with respect to the reference image, are $(0,0),(0,0.5),(0.5,0)$ and $(0.5,0.5)$, respectively. The high-pass filter, $\mathbf{C}$, is used to represent Laplacian kernel with dimension $5 \times 5$.

\section{Results an Discussions}

Figure 1, shows the reference phantom image (Fig. 1a) and its corresponding Sinogram (Fig. 1b). Figure 2 show the results of adopting the proposed algorithm with the simple back projection reconstruction algorithm. In Fig. 2a, zoomed parts of the reconstructed LR images is shown. The zoomed part of the reconstructed HR CT using proposed algorithm. From these figures, we can see that the reconstructed HR CT using proposed algorithm is sharper than the reconstructed LR image.

Also the results of adopting the filtered back projection with the proposed algorithm is shown in Fig. 3. Iin Fig. 2a, zoomed parts of the reconstructed LR images is shown. Iin Fig. 3b, the zoomed part of the reconstructed HR CT using proposed algorithm. From these figures, we can see that the reconstructed HR CT using proposed algorithm is sharper than the reconstructed LR image. Moreover, it can be shown that using filtered back projection is better than simple back projection, which is logic as stated in the litreature.

\section{CONCLUSION}

In this paper, we proposed a HR CT Reconstruction algorithm in the sinogram space. The proposed algorithm is solved SR reconstruction proplrm in th sinogram space rather than in the pixel domain. The conventional SR CT algorithms perform the enhancement in two steps, namely, reconstrut the CT image in pixel domain and then enhance the resolution by applying SR technique as a post-process. Based on the simulation results, the proposed algorithm enhances the resolution compared to the reconstructed LR CT image.

\section{REFERENCES}

[1] Thorsten M. Buzug, Computed Tomography From Photon Statistics to Modern Cone-Beam CT, Springer 2008.

[2] J. Hsieh et. al, Recent Advances in CT Image Reconstruction, Current Radiology Reports, Vol. 1, Issue 1, pp 39-51, March 2013.

[3] H. Greenspan, Super-Resolution in Medical Imaging, Computer Journal, vol. 52, No. 1, pp. 43-63, 2009.

[4] Ying Bai, Xiao Han, and Jerry L. Prince, Super-resolution Reconstruction of MR Brain Images, Proc. of 38-th Annual Conference on Information Sciences and Systems, Princeton, New Jersey, pp. 1358-1363, March 2004.

[5] Ali Gholipour, Simon K. Warfield, Super-resolution Reconstruction of Fetal Brain MRI, Workshop on Image Analysis for the Developing Brain, London, UK, pp. 45-52, September 2009.

[6] D. Kouamo and M. Ploquin, Super-resolution in medical imaging: An illustrative approach through ultrasound, IEEE International Symposium on Biomedical Imaging: From Nano to Macro, pp. 249 - 252, 2009.

[7] H. Tang, T. Zhuang and Ed X. Wu, Realizations of fast 2-D/3-D image filtering and enhancement, IEEE Transactions on Medical Imaging, vol. 20, no. 2, pp. 132-140, February 2008.

[8] G. M. Callic et. al, Analysis of fast block matching motion estimation algorithms for video Super-Resolution systems, IEEE Transactions on Consumer Electronics, vol. 54, issue 3, 1430 - 1438, 2008. 


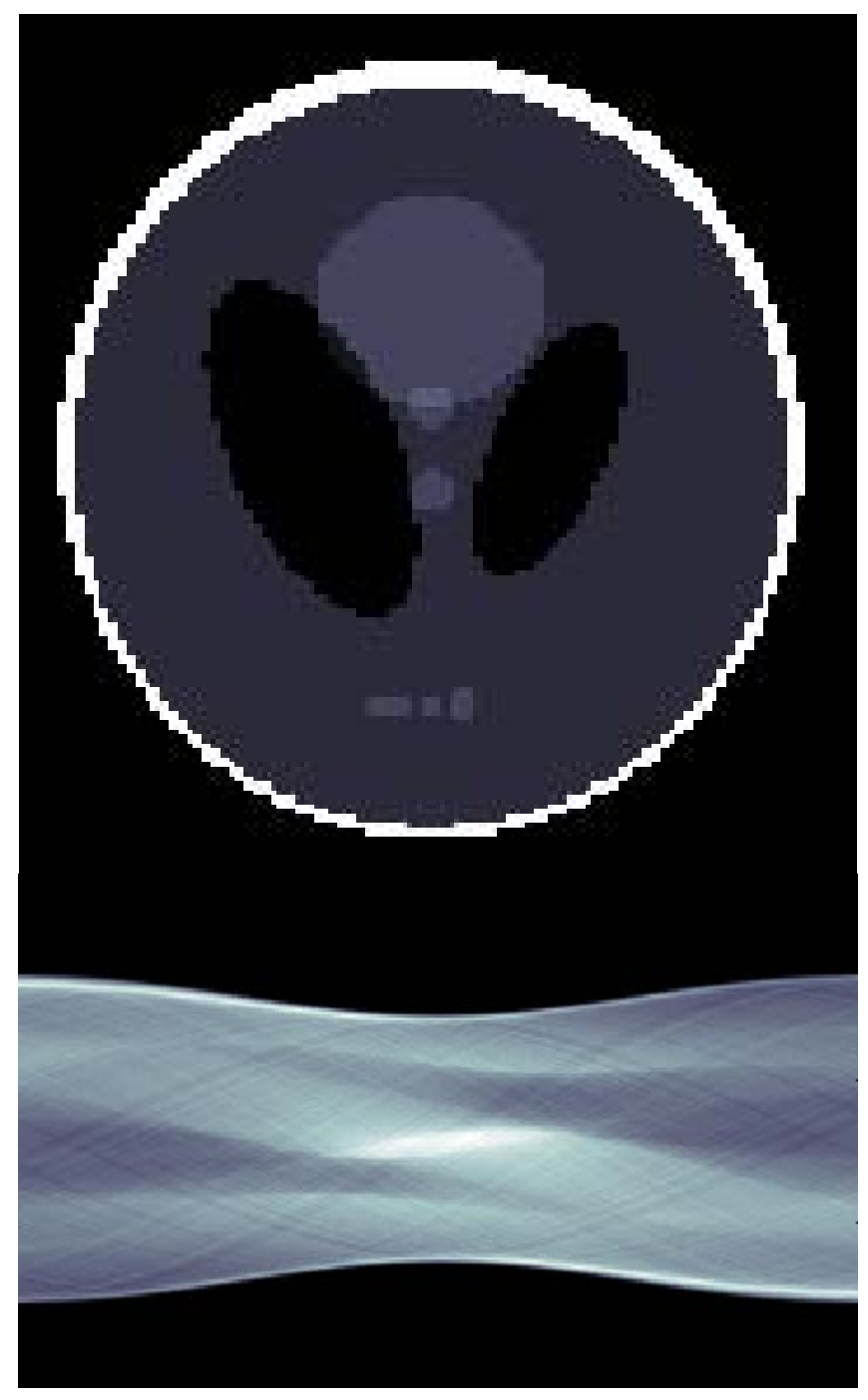

Fig. 1. From up to down, a) LR CT original Phantom image, b) The corresponding Sinogram space.

[9] S. Farsiu, M. D. Robinson, M. Elad, and P. Milanfar, Fast and Robust Multiframe Super Resolution, IEEE Trans. On Image Processing,vol. 13, no. 10 , Oct. 2004

[10] O. A. Omer and T. Tanaka, Region-based weighted-norm with adaptive regularization for resolution enhancement, Elsevier Digital Signal Processing, doi:10.1016/j.dsp. 2011.02.005.

[11] J. Anthony Parker, Robert V. Kenyon and Donald E. Troxel, Comparison of interpolating methods for image resampling, IEEE Transactions on Medical Imaging, vol. 2, no. 1, pp. 31-39, March 1993.

[12] K. P. Pruessmann, M. Weiger, M. B. Scheidegger, and P. Boesiger, SENSE: Sensitivity Encoding for Fast MRI, Magnetic Resonance in Medicine, Vol. 42, pp. 952-962, July 1999.

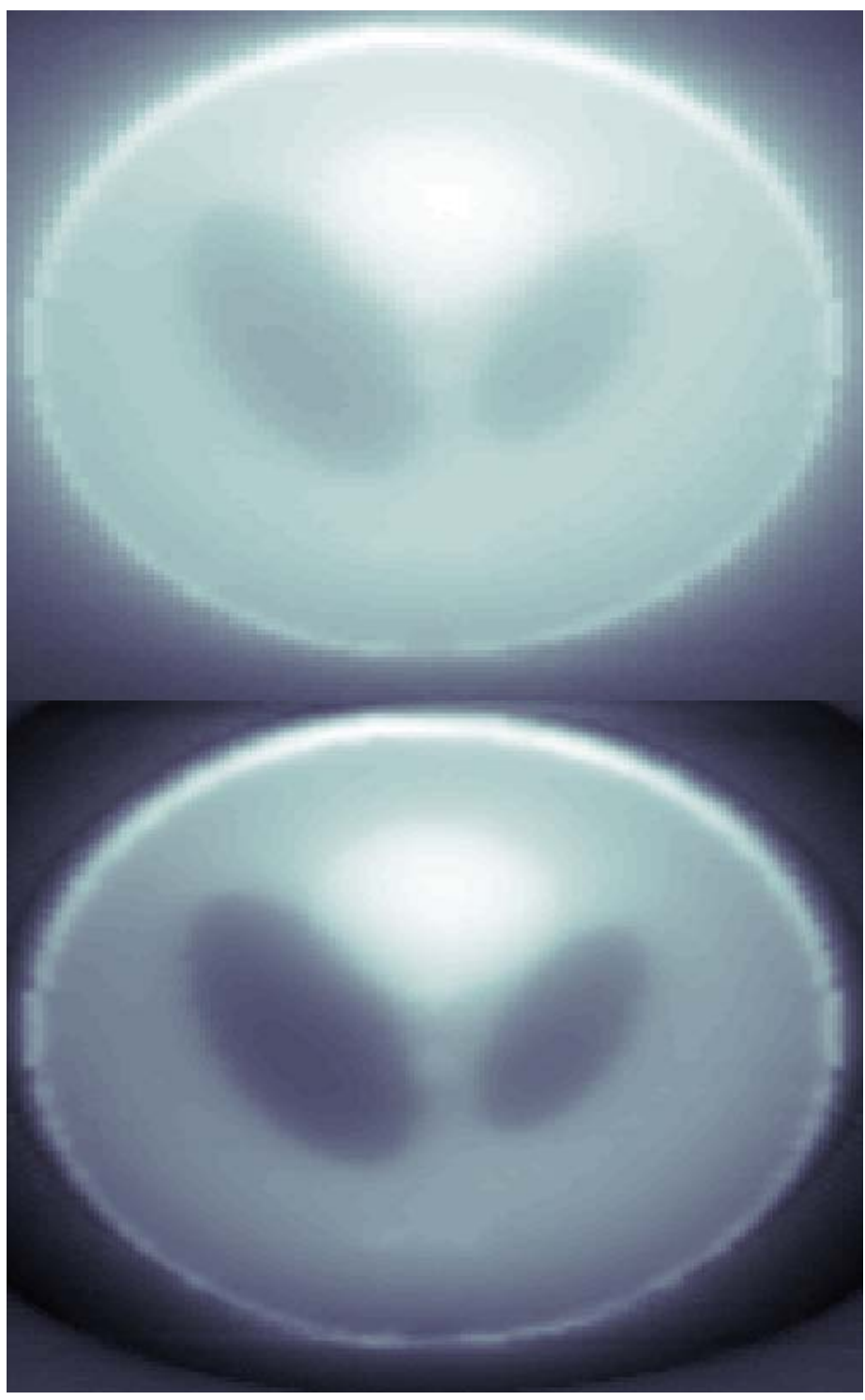

Fig. 2. From up to down, a) LR CT reconstructed phantom, b) result of HR CT reconstruction using simple back projection. 


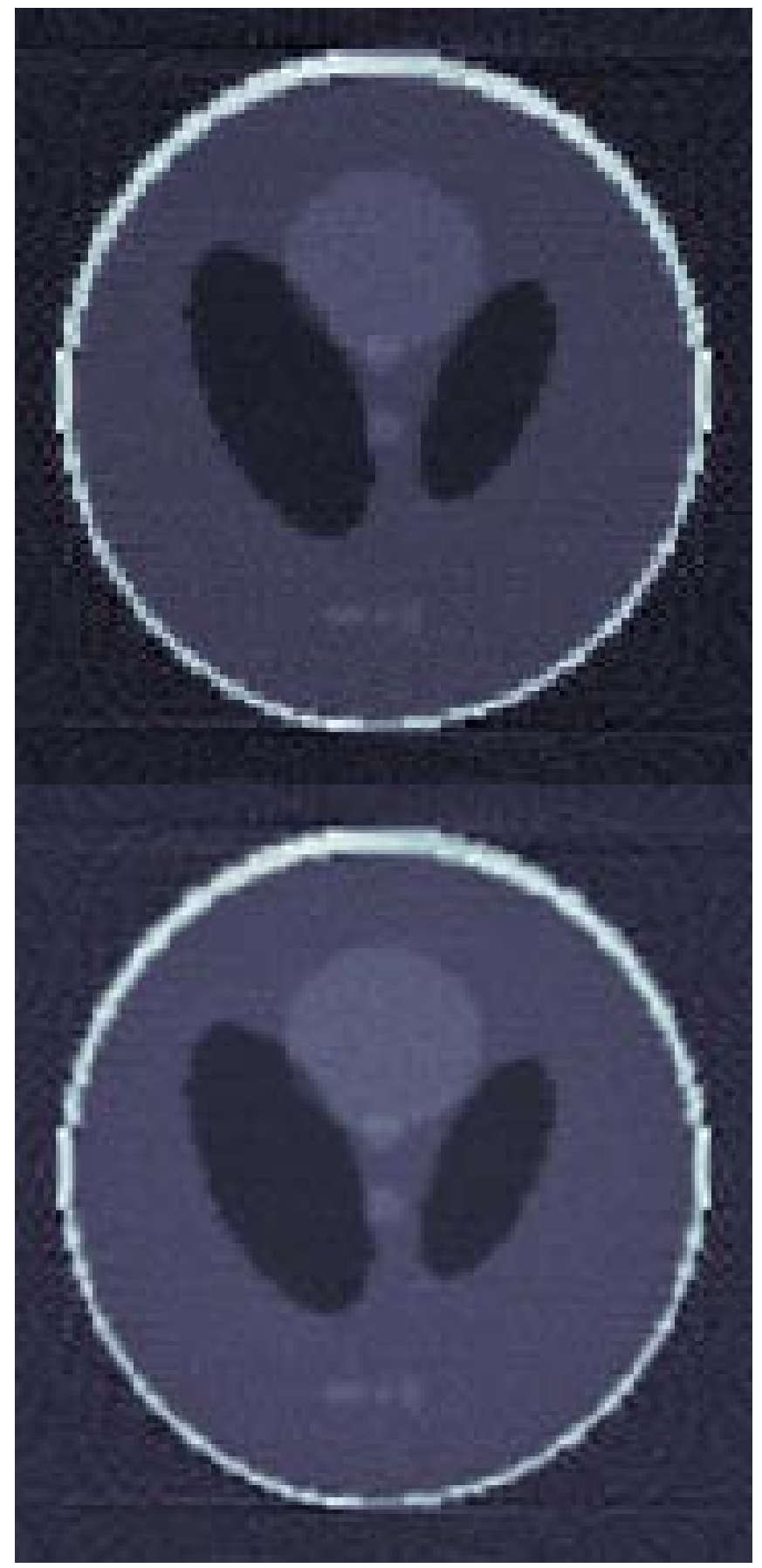

Fig. 3. From up to down, a) LR CT reconstructed phantom, b) result of HR $\mathrm{CT}$ reconstruction using filtered backprojection.

\section{Creative Commons Attribution License 4.0 (Attribution 4.0 International, CC BY 4.0)}

This article is published under the terms of the Creative Commons Attribution License 4.0

https://creativecommons.org/licenses/by/4.0/deed.en_US 